\title{
LOCALISED ELECTRON STATES AT THE INTERFACE BETWEEN TWO TRANSITION METALS
}

\author{
J. P. MUSCAT, M. LANNOO and G. ALLAN \\ Laboratoire de Physique des Solides $(*)$, \\ Institut Supérieur d'Electronique du Nord, 3, rue François-Baes, 59046 Lille Cedex, France
}

(Reçu le 26 octobre 1976, révisé le 31 janvier 1977, accepté le 2 février 1977)

\begin{abstract}
Résumé. - On étudie les états électroniques à l'interface cohérente de métaux de transition dans l'approximation des liaisons fortes. La bande d est représentée par une bande 5 fois dégénérée. Ceci permet une étude analytique de la possibilité d'existence d'états localisés au voisinage de l'interface. On suppose que ces conditions restent valables dans les cas d'une description plus exacte de la bande $d$.
\end{abstract}

\begin{abstract}
The electron states at coherent interfaces between transition metals are studied in a tight-binding approximation. The $d$ band is approximated by a fivefold degenerate $s$ band. This allows an analytical study of the possibility of existence of interface localised states. It is believed that the conditions which are derived should remain valid for the more complex case of $d$ bands.
\end{abstract}

1. Introduction. - The electronic properties of bimetallic interfaces have only begun to represent a serious topic of interest to metal physicists. This interest is due to the many metallurgical applications which could emanate from a knowledge of these properties. The present lack of knowledge must be attributed to the great difficulties encountered when the problem is tackled either from the theoretical or experimental points of view.

The experimental situation is rather complex in that the techniques which have proved so efficient in studying free surfaces or adsorbates cannot be generalized simply to study interfaces. Further the adsorption coverages were insufficient to constitute real interfaces. In fact one obtains a true interface only for coverages larger than three or four monolayers. For example this is obvious when one studies the variation of the work function during adsorption [1]. A constant limit value is obtained for such coverages.

From the theoretical viewpoint, only the crudest models can be set up to study the problem. These mainly deal with adhesional properties such as interface energies [2], or the electronic density difference introduced by the interface [3].

One technique which has proved extremely useful in studies of transition metal surfaces is the Green's

(*) Equipe de Recherche Associée au C.N.R.S.

LE JOURNAL DE PHYSIQUE. - T. 38, No 5, MAI 1977 function method [4,5] within the tight-binding approximation. This method has been used to determine the existence of surface states in cubic lattices [5-7]. We attempt here a generalization of these methods to study the analogous quantities for the bimetallic interface.

The problem may be treated by considering two semi-infinite metals having the same crystal structure, and whose surfaces are oriented in the same direction. This is already a rather drastic approximation, however going beyond this involves too much complexity. We shall also use the tight-binding approximation limited to nearest neighbours for an s-like band. This is certainly a less serious approximation, as equivalent models have shown that a good description of the properties of transition metals, can be obtained within this approximation [8].

The initial situation is represented schematically in figure $1 a$ where $\beta_{\mathrm{a}}$ and $\beta_{\mathrm{b}}$ are nearest neighbour interactions within each metal. This thus represents the unperturbed problem; the Green's functions for the cut crystal may be obtained analytically [5-6] for many possible orientations of the surface plane of the three different cubic lattices. The perturbation consists in bringing the two metals into contact to form the interface. Once put together, the two metals must have the same Fermi energy ; this can be obtained by the creation of a dipole layer $\Delta U$ at the interface (Fig. 1b). The final situation is illustrated in figure $1 c$. $\gamma$ represents the interaction between the two nearest 
a)

$\beta_{\mathrm{a}}$

b)

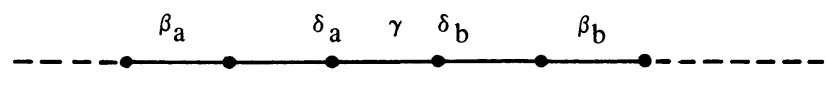

c)

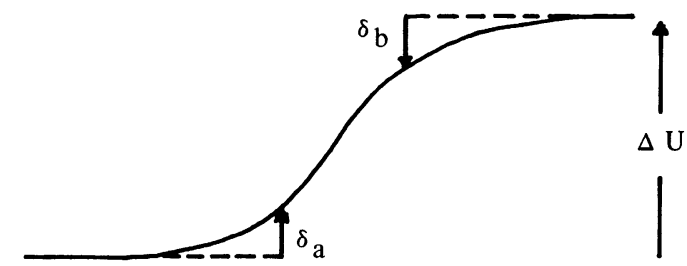

FIG. 1. $-a$ ) Schematic representation of metals $\mathrm{a}$ and $\mathrm{b}$ before bringing the two metals in contact. b) Selfconsistent potential. c) Metals $\mathrm{a}$ and $\mathrm{b}$ after contact.

neighbours on either side of the interface, $\delta_{\mathrm{a}}, \delta_{\mathrm{b}}$ and $\Delta U$ are potentials on the interface atoms which must be calculated self-consistently.

2. The Green's function method. - The Green's function $G$ of the perturbed system (Fig. 1c) may be expressed in terms of the Green's function $g_{0}$ of both semi-infinite metals (Fig. 1a). This is done by application of the Dyson equation

$$
G=g_{0}+g_{0} V G
$$

where $V$ is the perturbative potential.

This perturbing potential $V$ may be separated into two terms. The first one, which we call $U$, brings metal b to a constant potential $\Delta U$. The second one $U^{\prime}$ ensures the bond between metals $a$ and $b$ and also the differences between the true self-consistent potential and constant values of the potential inside metals a and $b$. Then equation (1) is identical to :

$$
\begin{aligned}
g & =g_{0}+g_{0} U g \\
G & =g+g U^{\prime} G
\end{aligned}
$$

The functions in our basis-set are Bloch-like with respect to translations parallel to the interface plane, but are localised within that plane [4]. This has the advantage of reducing the problem to that of a linear chain for a fixed $\mathbf{k}_{\|}$wave-vector [5-6]. We denote the $i$ th plane away from the surface of metal a by the subscript $i$ and superscript a. The non-zero matrix elements of $U$ are $U_{i i}^{\text {bb }}$ equal to $\Delta U$ for any value of $i$. As for the surface case or for any defect, we can assume that the perturbation potential is rapidly screened when one goes away from the interface plane. So the only non-zero elements of $U^{\prime}$ are

$$
\begin{aligned}
U_{00}^{\prime a a} & =\delta_{\mathrm{a}} \\
U_{00}^{\prime b b} & =\delta_{\mathrm{b}} \\
U_{00}^{\prime a b} & =\Gamma\left(\mathbf{k}_{\|}\right)
\end{aligned}
$$

where $\Gamma\left(\mathbf{k}_{\|}\right)$is the parallel Fourier transform of the $\gamma$ interactions. Within our approximations of a tightbinding s-band with only nearest-neighbour interactions, the matrix elements of $g_{0}, g$ and $G$ are analytical [5-6]. In the basis defined above, we call $E_{\mathrm{a}}\left(\mathbf{k}_{\|}\right)$ the diagonal term of the hamiltonian corresponding to a perfect infinite metal a, $V_{\mathrm{a}}\left(\mathbf{k}_{\|}\right)$the non diagonal term, $E_{\mathrm{b}}\left(\mathbf{k}_{\|}\right)$and $V_{\mathrm{b}}\left(\mathbf{k}_{\|}\right)$the corresponding terms for metal b. For example we get [5-7] :

$$
\begin{aligned}
g_{00}^{\mathrm{a}}(E)= & \frac{1}{2 V_{\mathbf{a}}^{2}\left(\mathbf{k}_{\|}\right)}\left\{\left[E-E_{\mathrm{a}}\left(\mathbf{k}_{\|}\right)\right]-\right. \\
& \left.-\left[\left(E-E_{\mathrm{a}}\left(\mathbf{k}_{\|}\right)\right)^{2}-4 V_{\mathbf{a}}^{2}\left(\mathbf{k}_{\|}\right)\right]^{1 / 2}\right\} \\
g_{00}^{\mathrm{b}}(E)= & \frac{1}{2 V_{\mathrm{b}}^{2}\left(\mathbf{k}_{\|}\right)}\left\{\left[E-E_{\mathrm{b}}\left(\mathbf{k}_{\|}\right)-\Delta U\right]-\right. \\
& \left.-\left[\left(E-E_{\mathrm{a}}\left(\mathbf{k}_{\|}\right)-\Delta U\right)^{2}-4 V_{\mathrm{b}}^{2}\left(\mathbf{k}_{\|}\right)\right]^{1 / 2}\right\} .
\end{aligned}
$$

We note that the potential $U$ corresponds only to the shift $\Delta U$ of the origin of the energies for metal b.

A knowledge of the Green's function $G$ gives us local densities of states for a given value of $\mathbf{k}_{\|}$. Straightforward calculations lead to :

$$
\begin{aligned}
G_{i i}^{\mathrm{aa}}= & g_{i i}^{\mathrm{a}}+ \\
& +\frac{g_{0 \mathrm{i}}^{\mathrm{a}} g_{i 0}^{\mathrm{b}}\left[\delta_{\mathrm{a}}+g_{00}^{\mathrm{b}}\left(\Gamma^{2}-\delta_{\mathrm{a}} \delta_{\mathrm{b}}\right)\right]}{\left(1-g_{00}^{\mathrm{a}} \delta_{\mathrm{a}}\right)\left(1-g_{00}^{\mathrm{b}} \delta_{\mathrm{b}}\right)-\Gamma^{2} g_{00}^{\mathrm{a}} g_{00}^{\mathrm{b}}}
\end{aligned}
$$

A numerical integration over $\mathbf{k}_{\|}$would give us the atomic local densities of states. However, it might prove more worthwhile at present to draw qualitative conclusions of physical interest rather than to give an accurate lengthy quantitative description in such a simple band model. In this respect, an interesting property to study is the existence of localised states at the interface. These exist if there are solutions of the equation :

$$
\begin{aligned}
{\left[1-\delta_{\mathrm{a}} g_{00}^{\mathrm{a}}(E)\right]\left[1-\delta_{\mathrm{b}} g_{00}^{\mathrm{b}}(E)\right]-} & \\
& -\Gamma^{2} g_{00}^{\mathrm{a}}(E) g_{00}^{\mathrm{b}}(E)=0
\end{aligned}
$$

for values of $E$ which lie outside the bands.

But before solving this equation, we must calculate the potentials $\delta_{\mathrm{a}}$ and $\delta_{\mathrm{b}}$ self-consistently. In order to do this, we need two self-consistent conditions. The first of these might be that the Friedel sum rule be satisfied, i.e. that the total displaced electronic charge at the Fermi level $\Delta N$ be zero, where $\Delta N$ is given by :

$$
\begin{array}{r}
\Delta N=-\frac{1}{\pi} \iint \arg \{[ \\
\left.-\delta_{\mathrm{a}} g_{00}^{\mathrm{a}}(E)\right]\left[1-\delta_{\mathrm{b}} g_{00}^{\mathrm{b}}(E)\right]- \\
\left.-\Gamma^{2} g_{00}^{\mathrm{a}}(E) g_{00}^{\mathrm{b}}(E)\right\} \mathrm{d}_{\|}
\end{array}
$$

where the integral is over the surface Brillouin zone.

The second condition is provided by the condition that the calculated dipole layer arising from the perturbation be equal to the initial dipole layer (i.e. the difference in Fermi energies of the two metals [10]). In order to calculate this quantity, we need to know the electronic charge difference on each plane; this 
represents an extremely difficult numerical problem. The charge oscillations rapidly decrease as one moves away from the interface plane. Let us assume that charge transfers occur only on the atom lying in planes which are nearest neighbours of the interface. We call $\delta N$ the net charge of atoms in plane (0) of metal (a) (Fig. 1). Then the Friedel sum rule leads to an opposite charge $(-\delta N)$ on each atom of plane $(0)$ of metal (b). Classical electrostatics gives the corresponding dipole height

$$
\Delta \varphi=\frac{4 \pi d}{S} \delta N
$$

where $d$ is the distance between planes and $S$ the area of the unit cell. Since $4 \pi d / S$ is of the order of $50 \mathrm{eV}$ per electron [9], we see that any error in $\delta N$ would be magnified considerably for $\Delta \varphi$.

The initial dipole layer is equal to the difference between the Fermi energies of metals $a$ and $b$. In the tight-binding approximation, if we call $E_{\mathrm{F}}^{\mathrm{i}}$ the Fermi level of metal $i(i=a$ or $b)$ referred to the bulk atomic level $E_{\mathrm{d}}^{\mathrm{i}}$, we get :

$$
\begin{aligned}
\Delta U & =\left(E_{\mathrm{F}}^{\mathrm{b}}+E_{\mathrm{d}}^{\mathrm{b}}\right)-\left(E_{\mathrm{F}}^{\mathrm{a}}+E_{\mathrm{d}}^{\mathrm{a}}\right) \\
& =\left(E_{\mathrm{F}}^{\mathrm{b}}-E_{\mathrm{F}}^{\mathrm{a}}\right)+\left(E_{\mathrm{d}}^{\mathrm{b}}-E_{\mathrm{d}}^{\mathrm{a}}\right) .
\end{aligned}
$$

The first term of $(8 b)$ may be calculated if one knows the bulk densities of states. Then one can assume that the difference between the bulk atomic levels and the free atom levels are equal. We have shown [10] that the change of the repulsion of the $d$ electrons by the $s$ electrons is close to $4 \mathrm{eV}$ for any transition metal.

The dipole layer $\Delta U$ is small, of the order of $1 \mathrm{eV}$, and so $\delta N$ is of the order of one hundredth. It does therefore appear more reasonable not to pursue these lines, but rather to determine the self-consistency, by an approximative method which would short-circuit the numerical difficulties. For instance an appropriate approximative method is the method of Haydock et al. [11]. Once self-consistency is achieved, we shall return to the Green's function method introduced above.

3. Self-consistency. - The method of Haydock et al. [11] is an interpolation scheme which is useful in determining local density of states. We shall apply this scheme in its simplest form, i.e. restricted to the 2nd moment approximation. The Green's function on an interface atom is then simply

$G(E)=\frac{1}{2 b_{1}}\left\{E-a_{1} \pm\left[\left(E-a_{1}\right)^{2}-4 b_{1}\right]^{1 / 2}\right\}$

where $a_{1}$ is the first order moment $a_{1}=\delta_{\mathrm{a}}$ or $\delta_{\mathrm{b}}$, and $b_{1}$ is the second order moment and is thus a summation over all nearest neighbours of the atom; expressions for $b_{1}$ of course depend on the orientation of the surface planes, and the crystal structure of the metals.

For a bulk atom (an atom other than the surface atom), we have $a_{1}=0, b_{1}=n \beta^{2}$ where $n$ is the number of nearest neighbours in the crystal, and $\beta$ the nearest neighbour interaction.

From these, we can easily calculate density on any atom. This is given by :

$$
\begin{aligned}
N=\frac{1}{2}+ & \frac{1}{\pi}\left\{\arcsin \left(\frac{E_{\mathrm{F}}-a_{1}}{2 b_{1}^{1 / 2}}\right)+\right. \\
& \left.+\frac{E_{\mathrm{F}}-a_{1}}{4 b_{1}}\left[4 b_{1}-\left(E_{\mathrm{F}}-a_{1}\right)^{2}\right]^{1 / 2}\right\}
\end{aligned}
$$

where the $E_{\mathrm{F}}$ 's are measured from the centre of the band.

Now it is straightforward to apply the self-consistency conditions in order to calculate $\delta_{\mathrm{a}}$ and $\delta_{\mathrm{b}}$. This can be done numerically. The calculations performed indicate that the self-consistent electronic density difference $\Delta N_{\mathrm{i}}$ between an interface atom, and a bulk atom is negligible, in all cases. This leads us to consider the small $\Delta N_{\mathrm{i}}$ limit where it is easily shown that :

$$
\delta_{\mathrm{i}}=E_{\mathrm{Fi}}\left(1-\frac{\left(b_{1}^{\mathrm{i}}\right)^{1 / 2}}{n^{1 / 2} \beta_{\mathrm{i}}}\right)+\sigma\left(\Delta N_{\mathrm{i}}\right)
$$

where $\mathrm{i}=\mathrm{a}$ or $\mathrm{b}$ and $\sigma\left(\Delta N_{\mathrm{i}}\right)$ stands for a term which is of the order of $\Delta N_{i}$ divided by the density of states at the Fermi level.

We have shown above that $\Delta N_{\mathrm{i}}$ is of the order of 0.01 and as the density of states for a transition metal is of the order of 1 state/eV, one can easily see that the first order term in $\Delta N_{\mathrm{i}}$ is negligible as soon as $b_{1}^{\mathrm{i}}$ differs from the bulk value $n \beta_{i}^{2}$. The same model applied to transition metal surfaces [9] gives a good description of the $\delta_{\mathrm{i}}$ behaviour along a transition series.

Clearly $b_{1}$ is going to differ from $n \beta^{2}$ if :

(i) the interaction $\gamma$ between atoms from different metals is different to $\beta_{\mathrm{i}}$,

(ii) there are many nearest neighbours of the interface atoms which are atoms of the other metal.

Hence we see that $\delta_{\mathrm{i}}=0$ if $\gamma=\beta_{\mathrm{i}}$, and $\delta_{\mathrm{i}}$ is greatest for cases such as that of two body-centered-cubic metals of different transition series whose surfaces are oriented in the (100) directions in which case we have

$$
\begin{aligned}
\gamma & =\left(\beta_{\mathrm{a}} \beta_{\mathrm{b}}\right)^{1 / 2} \\
b_{1}^{\mathrm{i}} & =4 \beta_{\mathrm{i}}^{2}+4 \gamma^{2} .
\end{aligned}
$$

From this treatment, we see that the self-consistent parameters can have the same sign for instance for the case of two transition metals of different series, whose Fermi energies have different signs. This would mean that an oscillation of the self-consistent potential appears on one side of the interface.

4. Existence of localised states. - Localised states exist if there are solutions to equation (6) for values of $E$ lying outside the energy bands.

Let us look briefly at some simple limits, such as for instance the non self-consistent situation $\delta_{\mathrm{a}}=\delta_{\mathrm{b}}=0$. 
Then it is easily shown that there can be no localised states in this case. Indeed equation (6) reduces (if we drop subscript 0 ) to :

$$
1-\Gamma^{2} g_{\mathrm{a}}(E) g_{\mathrm{b}}(E)=0
$$

where $\Gamma=\left(V_{\mathrm{a}} V_{\mathrm{b}}\right)^{1 / 2}$.

From equation (4), it is easily seen that $g_{\mathrm{a}}(E)$ and $g_{\mathrm{b}}(E)$ are decreasing functions of $E$ for values of $E$ outside the bands. We must therefore have

$$
\left|V_{\mathrm{a}} g_{\mathrm{a}}(E)\right|<\left|V_{\mathrm{a}} g_{\mathrm{a}}\left(E_{\mathrm{a}} \pm 2 V_{\mathrm{a}}\right)\right|=1
$$

for $\left|E-E_{\mathrm{a}}\right|>2 V_{\mathrm{a}}$, and

$$
\left|V_{\mathrm{b}} g_{\mathrm{b}}(E)\right|<\left|V_{\mathrm{b}} g_{\mathrm{b}}\left(E_{\mathrm{b}}+\Delta U \pm 2 V_{\mathrm{b}}\right)\right|=1
$$

for $\left|E-E_{\mathrm{b}}-U\right|>2 V_{\mathrm{b}}$.

This means that (14) cannot be satisfied for values of $E$ outside the bands, i.e. that there is no possibility of localised states in the non self-consistent case. Self-consistency must thus be included. However it was shown that the self-consistent potentials $\delta_{\mathrm{a}}$ and $\delta_{\mathrm{b}}$ were generally small and that they should not play a predominant role in the existence of localised states except in the most favourable cases. These are the cases where $\delta_{\mathrm{a}}$ and $\delta_{\mathrm{b}}$ are non-negligible as compared to $V_{\mathrm{a}}$ and $V_{\mathrm{b}}$. Now $V_{\mathrm{a}}$ and $V_{\mathrm{b}}$ represent the bandwidths at given $\mathbf{k}_{\|}$, in some cases these could be equal to zero for certain points of the surface Brillouin zone; e.g. for the (100) face of a body centred cubic lattice we have [1]

$$
\left.\begin{array}{l}
V_{\mathbf{i}}\left(\mathbf{k}_{\|}\right)=4 \beta_{\mathbf{i}} \cos \left(\frac{k_{x} a}{2}\right) \cos \left(\frac{k_{y} a}{2}\right) \\
E_{\mathbf{i}}\left(\mathbf{k}_{\|}\right)=0
\end{array}\right\} .
$$

We thus see that $V_{\mathrm{i}}=0$ when $k_{x} a$ or $k_{y} a$ are equal to $\pi$ or $-\pi$. Let us look at this simple limit briefly. The real parts of the Green's functions are given by :

$$
\begin{aligned}
& g_{\mathrm{a}}(E)=\frac{1}{E} \\
& g_{\mathrm{b}}(E)=\frac{1}{E-\Delta U}
\end{aligned}
$$

Then equation (6) reduces to

$E(E-\Delta U)-\delta_{\mathrm{a}}(E-\Delta U)-g_{\mathrm{b}} E+\delta_{\mathrm{a}} \delta_{\mathrm{b}}=0$.

The solutions of which are

$$
\left.\begin{array}{l}
E_{1}=\delta_{\mathrm{a}} \\
E_{2}=\Delta U+\delta_{\mathrm{b}}
\end{array}\right\} .
$$

Now we have clearly localised states since $\delta_{\mathrm{a}}$ and $\delta_{\mathrm{b}}$ are non-zero. The cases of greatest interest are those for which $\Delta U$ is large, i.e. two metals on either side of $E_{\mathrm{F}}=0$; then solving for $\delta_{\mathrm{a}}$ and $\delta_{\mathrm{b}}$, we find that these must have the same sign. This means that both the original levels at 0 and $\Delta U$ are shifted in the same direction (see Fig. 2). Hence we must have a localised state between the levels (provided of course $\delta_{\mathrm{a}}<\Delta U$ ) and one above the highest level.



Fig. 2. - Relative value of $\delta_{\mathrm{a}}, \delta_{\mathrm{b}}$ when $\Delta U$ is large $\left(E_{\mathrm{F}}^{\mathrm{a}}<0, E_{\mathrm{F}}^{\mathrm{b}}>0\right)$.

Making the bandwidths finite of course complicates matters somewhat, and we can no longer give an exact description in analytical form. However we can use certain approximations in order to simulate reality simply and not too imprecisely. We have already said that the most favourable cases for the existence of localised states are those for which we have two widely different metals, one from the beginning of the first series, and the other from the end of the third series. This gives us a fairly wide seperation of the band centre e.g. in the cases $N_{\mathrm{a}}=2, N_{\mathrm{b}}=8$, $2 \beta_{\mathrm{a}}=1.25 \mathrm{eV}, 2 \beta_{\mathrm{b}}=1.75 \mathrm{eV}$, we find $\Delta U=4.176 \mathrm{eV}$. The self-consistent values of the potentials are equal to $\delta_{\mathrm{a}}=0.174 \mathrm{eV}, \delta_{\mathrm{b}}=0.244 \mathrm{eV}$ for the (100) face of two b.c.c. metals.

Now in order to have localised states, we need to have narrow bands, i.e.

$$
V_{\mathrm{i}} \ll \Delta U \text {. }
$$

This would mean that the bands be widely separated (see Fig. 3).

Let us now consider the quantity

$$
\begin{aligned}
F(E)=\delta_{\mathrm{a}} g_{\mathrm{a}}(E) & +\delta_{\mathrm{b}} g_{\mathrm{b}}(E)+ \\
& +\left(V_{\mathrm{a}} V_{\mathrm{b}}-\delta_{\mathrm{a}} \delta_{\mathrm{b}}\right) g_{\mathrm{a}}(E) g_{\mathrm{b}}(E)
\end{aligned}
$$

now $F(E)$ is negative for $E<-2 V_{\mathrm{a}}$. This is so because both $\delta_{\mathrm{a}}$ and $\delta_{\mathrm{b}}$ are positive, and both $g_{\mathrm{a}}(E)$ and $g_{\mathrm{b}}(E)$ negative in this range of $E$. This necessarily means that there can be no roots of $1-F(E)=0$, in this energy range.

In the region between the bands

$$
2 V_{\mathrm{a}}<E<\Delta U-2 V_{\mathrm{b}}
$$

we must have $g_{\mathrm{a}}(E)>0$ and $g_{\mathrm{b}}(E)<0$; in order to have a localised state we must have as large a value 


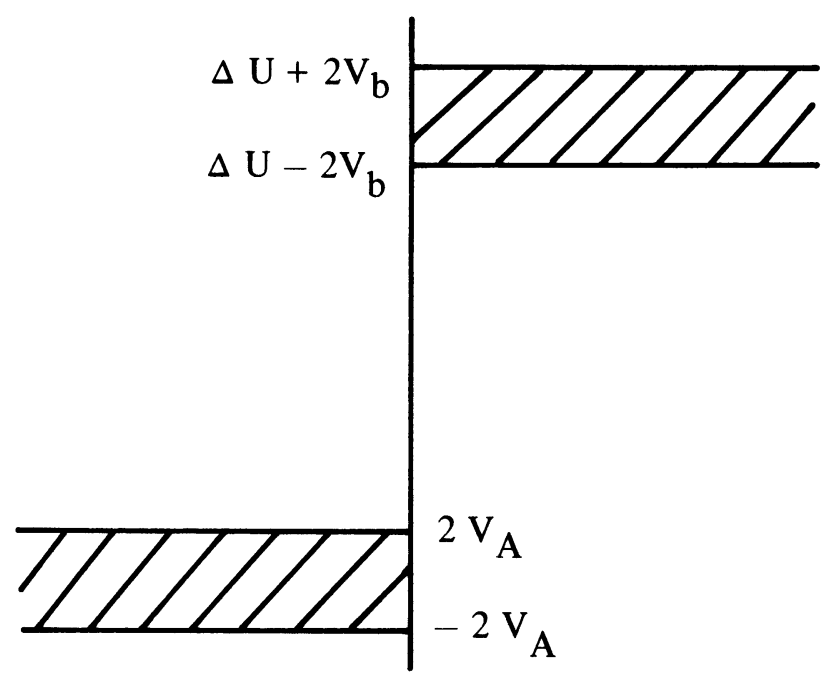

FIG. 3. - Schematic representation of the bands on either side of the interface $\left(V_{\mathrm{a}} \simeq V_{\mathrm{b}} \ll \Delta U\right)$.

of $\left|g_{\mathrm{a}}(E)\right|$ and as small a value of $\left|g_{\mathrm{b}}(E)\right|$ as possible. The best possibility is for $E=2 V_{\mathrm{a}}$, then

$$
\begin{gathered}
g_{\mathrm{a}}(E)=\frac{1}{V_{\mathrm{a}}} \\
g_{\mathrm{b}}(E)=\frac{1}{2 V_{\mathrm{b}}^{2}}\left\{2 V_{\mathrm{a}}-\Delta U+\left[\left(2 V_{\mathrm{a}}-U\right)^{2}-4 V_{\mathrm{b}}^{2}\right]^{1 / 2}\right\}= \\
=-\frac{1}{\Delta U}+0\left(\frac{V_{\mathrm{i}}}{\Delta U}\right)
\end{gathered}
$$

localised states hence exist if :

$$
\frac{\delta_{\mathrm{a}}}{V_{\mathrm{a}}}-\frac{V_{\mathrm{b}}}{\Delta U} \frac{1}{1+\frac{\delta_{\mathrm{b}}}{\Delta U}}>1 .
$$

One can immediately see that a necessary but nonsufficient condition is given by :

$$
\frac{\delta_{\mathrm{a}}}{V_{\mathrm{a}}}>1
$$

If we now replace $\delta_{\mathrm{a}}$ and $\beta_{\mathrm{a}}$ by the calculated values, we see that localised states exist between the bands for values of $\mathbf{k}_{\|}$which satisfy the inequality

$$
\cos \left(\frac{k_{x} a}{2}\right) \cos \left(\frac{k_{y} a}{2}\right)<0.07 \text {. }
$$

The region of $\mathbf{k}_{\|}$space in the surface Brillouin zone for which there are localised states is thus a thin region at the edge of the zone (see Fig. 4). We finally look at the region above the highest band, i.e. $E>U+2 V_{\mathrm{b}}$. Now both Green's functions are positive and so they should both contribute to $F(E)$. Hence this is the region where localised states are most likely. However the most significant contribution will arise from $g_{\mathrm{b}}(E)$. The condition for localised states to exist in this

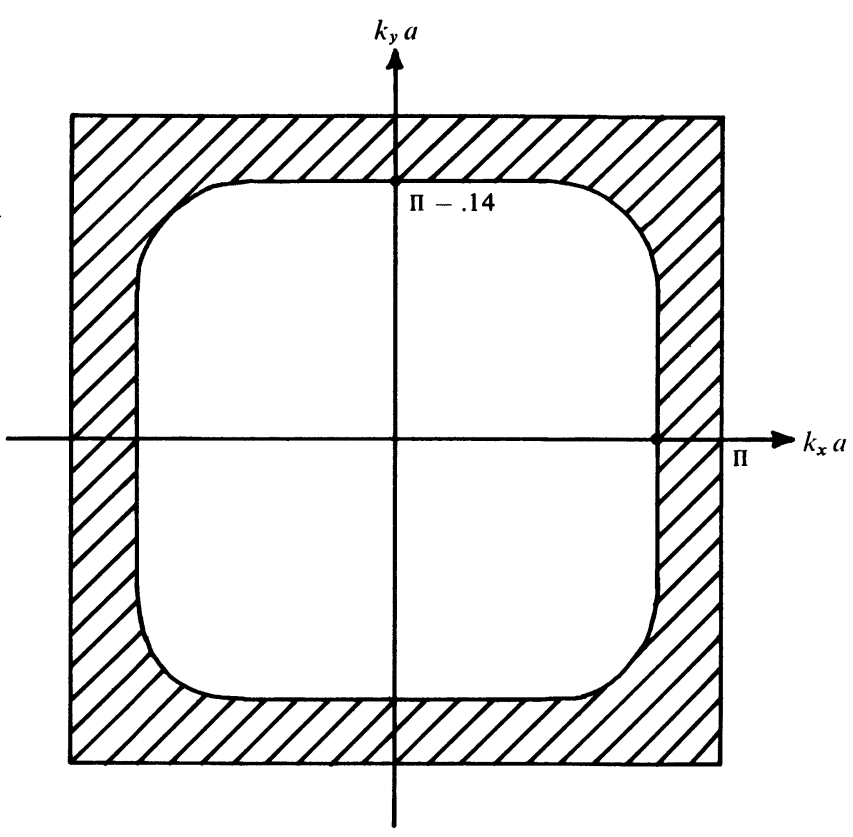

FIG. 4. - Region in the (100) surface Brillouin zone of a b.c.c. crystal for which there are localised states.

energy range within the approximation $V_{\mathbf{i}} \ll U$ is now given by

$$
\frac{\delta_{\mathrm{b}}}{V_{\mathrm{b}}}+\frac{V_{\mathrm{a}}}{\Delta U} \frac{1}{1-\frac{\delta_{\mathrm{a}}}{\Delta U}}>1
$$

Finally replacing all parameters by the calculated values, we see that localised states exist above the highest band, for values of $\mathbf{k}_{\|}$which satisfy the inequality

$$
\cos \left(\frac{k_{x} a}{2}\right) \cos \left(\frac{k_{y} a}{2}\right)<0.09
$$

This gives us a region in the surface Brillouin zone which is slightly more extended than in the previous case.

5. Conclusion. - We have thus shown that states localised at an interface between two transition metals can exist within our model, for certain favourable cases. The most favourable cases are furnished by two very different metal e.g. Sc and $\mathrm{Pt}$, or $\mathrm{Ni}$ and $\mathrm{Lu}$. These are f.c.c. metals, and we only considered b.c.c. metals in our model, however the situation would not be too different for f.c.c. metals. If we choose the (100) face, then we know that four of the twelve nearest neighbours of an interface atom will be atoms from the other metal. This would give us values of the secondorder moment still different from the bulk secondorder moment, and from (13) we should still have nonnegligible though smaller values of the potentials $\delta_{\mathrm{a}}$ and $\delta_{\mathrm{b}}$. We know also [9] that $V_{\mathrm{i}}$ is equal to zero at the limit of the surface Brillouin zone $\left(k_{x} a+k_{y} a=2 \pi\right)$. 
The treatment for two f.c.c. metals is slightly more complicated, however the results should not be changed too much.

Experimental observation of these localised states is still very much a hypothetical question at the present time. However the photo-emission escape depth seems to be just sufficient to see the properties of the interface metal-adsorbates when the coverage is equal to three or four monolayers. As for grain boundaries, these localised states and in general the electronic structure near the interface have some influence on the electrical properties of the interface. A better knowledge of the atomic structure near the interface would be necessary to study such effects. However a theoretical prediction is by no means uninteresting as it might encourage experimentalists to study these systems. Let us note further that the present model represents only a first step towards a much more complete analysis of these systems, but the features we have described should still be present in a more refined model using a d-basis set. Work using a more realistic d-band is in progress.

\section{References}

[1] Leynaud, M., Allan, G., Surf. Sci. 53 (1975) 359; LeyNAUD, M., Thèse 3 e Cycle Lille (1975).

[2] Djafari-Rouhani, M. and Schuttler, R., Surf. Sci. 38 (1973) 503.

Ferrante, J. and Smith, J. R., Surf. Sci. 38 (1973) 77.

VANNimenus, J. and BuDd, H. F., to be published.

Allan, G., Lannoo, M. and Dobrzynski, L., Phil. Mag. 30 (1974) 33.

[3] Bennett, A. J. and Duke, C. B., Phys. Rev. 160 (1967) 541.

MusCat, J. P. and Allan, G., to be published.

[4] Brown, R. A., Phys. Rev. 156 (1967) 889.

[5] Allan, G., Ann. Phys. 5 (1970) 169.

[6] Kalkstein, D. and Soven, P., Surf. Sci. 26 (1971) 85.

[7] Allan, G. and Lenglart, P., Surf. Sci. 30 (1972) 641.
[8] Friedel, J., The Physics of Metals (C.U.P.) 1969.

[9] Allan, G., Electronic structure of transition metals, to be published in Surface properties, surface states of materials, M. Dekker, New York;

ALLAN, G., Electronic structure and reactivity of metal sursurfaces, NATO Advanced Study Institutes Series, E. G. Derouane and A. A. Lucas Ed. (Plenum Press N.Y.) 1976, p. 45-81.

[10] Allan, G., Lannoo, M., Second Colloque de Physique et Chimie des Surfaces (Brest, mai 1975); Le Vide, Les Couches Minces, 30A (1975) 1 ; Vide 30 (1975) 48.

[11] Haydock, R., Heine, V. and Kelly, M. J., J. Phys. C 5 (1973) 2845 . 